\title{
THE REPRESENTATION OF CHAINABLE CONTINUA WITH ONLY TWO BONDING MAPS
}

\author{
SAM W. YOUNG
}

Definition. A set $S$ of continuous functions of $[0,1]$ into $[0,1]$ is called a "complete" set of bonding maps if every chainable continuum can be obtained as the inverse limit of an inverse mapping system each of whose coordinate spaces is $[0,1]$ and each of whose bonding maps is in $S$.

It is shown in [1] that a dense set is complete. Jolly and Rogers in [3] demonstrate a complete set with only four elements. It follows from the theorem of Jarník and Knichal [2] that there is a complete set with only two elements. Mahavier proves in [4] that every complete set must have at least two elements and therefore two is the minimum number.

In this note, we prove the following:

THEOREM. There exists a continuous function $f$ of $[0,1]$ into $[0,1]$ such that $\left\{f, \frac{1}{2} f\right\}$ is complete.

First we will establish a lemma, the proof of which closely follows [2]. Let $C$ denote the set of all continuous functions of $[0,1]$ into $[0,1] . e(x)=\frac{1}{2} x, e^{n+1}(x)=e\left(e^{n}(x)\right)$ and $e^{0}(x)=x$.

Lemma. If $\theta_{1}, \theta_{2} \in C$, then there exists $g \in C$ such that gege $e^{3}=\theta_{1}$ and $g e^{2} g e^{3}=\theta_{2}$.

Proof. Let $g$ be defined as follows: $g(x)=3 \cdot 2^{-2}+2 x$ for $0 \leqq x \leqq 2^{-3}$, $g(x)=\theta_{2}(16 x-3)$ for $3 \cdot 2^{-4} \leqq x \leqq 2^{-2}, g(x)=\theta_{1}(8 x-3)$ for $3 \cdot 2^{-3} \leqq x$ $\leqq 2^{-1}$, and $g$ is linear in each of the intervals $\left[2^{-3}, 3 \cdot 2^{-4}\right],\left[2^{-2}, 3 \cdot 2^{-3}\right]$, and $\left[2^{-1}, 1\right]$. It is now easy to verify that $g e g e^{3}=\theta_{1}$ and $g e^{2} g e^{3}=\theta_{2}$.

PROOF OF THE THEOREM. It follows from the lemma and a remark made previously that there exists $g \in C$ such that $\left\{g e g e^{3}, g e^{2} g e^{3}\right\}$ is complete. Thus if $M$ is a chainable continuum, then $M$ can be represented in the form $M \cong g e^{n_{1}} g e^{3} g e^{n_{2}} g e^{3} g e^{n_{3}} g e^{3} g e^{n_{4}} g e^{3} \ldots$. The diagram arrows are omitted to save space. Each of the exponents $n_{i}$ is 1 or 2 . Let $f=e g e^{3} g$ and then by regrouping the bonding maps and deleting the first map, we obtain $M \cong e^{n_{1}-1} f e^{n_{2}-1} f e^{n_{3}-1} f \cdots$. Now for each $i$, $n_{i}-1=0$ or 1 and so another regrouping yields $M \cong\left(a_{1} f\right)\left(a_{2} f\right)$ $\left(a^{3} f\right) \cdots$ where for each $i, a_{i}=1$ or $\frac{1}{2}$. This completes the proof.

Received by the editors January 17, 1969. 
It should be noted that $\left\{g e g e^{3}, g e^{2} g e^{3}\right\}$ is complete as a consequence of the fact that the collection of all finite compositions of these functions is dense in $C$. The collection of all finite compositions of the functions $f$ and $\frac{1}{2} f$ is not dense in $C$ since the range of $f$ is a proper subinterval of $[0,1]$.

\section{REFERENCES}

1. Morton Brown, Some applications of an approximation theorem for inverse limits, Proc. Amer. Math. Soc. 11 (1960), 478-483.

2. V. Jarník and V. Knichal, Sur l'approximation des fonctions continues par les superpositions de deux fonctions, Fund. Math. 24 (1935), 206-208.

3. R. F. Jolly and J. T. Rogers, Jr., Inverse limit spaces defined by only finitely many distinct bonding maps, Fund. Math. (to appear)

4. William S. Mahavier, A chainable continuum not homeomorphic to an inverse limit on $[0,1]$ with only one bonding map, Proc. Amer. Math. Soc. 18 (1967), 284-286.

UNIVERSITY OF UTAH 